\title{
V6: Projekt Lernendenstation
}

\section{Doris Wilborn}

Online publiziert: 23. Oktober 2013

(C) Springer-Verlag Wien 2013

Einleitung: Die Studierenden übernehmen alle Aufgaben von Pflege in Eigenverantwortung, in verantwortlicher Mitwirkung und in interdisziplinärer Zusammenarbeit in der Lernendenstation. Die Studierenden vertiefen im Projekt ihre im Studium bereits erworbenen Handlungs- und Reflexionskompetenzen und erwerben spezielle neue Handlungskompetenzen.

Forschungsfrage: Inwiefern ist eine Veränderung der Kompetenzen durch den Einsatz in der Lernendenstation erfolgt? Methode: 27 Studierende nahmen an der Kompetenzmessung teil. Es erfolgte eine der Kompetenzen im pre-post-Design.

Die Lernendenstation wurde in einer rehabilitativ-geriatrischen Station durchgeführt.

Es wurde ein Fragebogen entwickelt, der die Dimensionen Übergeordnete Aspekte, Pflegeprozess, Kommunikation und Individuelle Unterstützung in der Selbstversorgung der Patienten enthält. Das Kompetenzverständnis, das dem Fragebogen zugrunde liegt, bezieht sich auf den Qualifikationsbegriff von Darmann und Reuschenbach (2013).
Es werden die Gesamtwerte der Kompetenzen, basierend auf Mittelwert-Berechnungen, vor und nach dem Einsatz berechnet, um mögliche Kompetenzveränderungen sichtbar zu machen. Unterschiede in der Kompetenzeinschätzung vor und nach dem Projekt werden mittels T-Test für verbundene Stichproben berechnet.

Ergebnisse: Von 24 Studierenden liegen die vollständig ausgefüllten Fragebogen beider Datenerhebungen vor. Die Selbsteinschätzung des Gesamtwissens zeigt eine Erhöhung des Scores nach Abschluss der Lernendenstation. Auch im Bereich der Einschätzung des Könnens liegt der Gesamtwert nach der Lernendenstation höher. Im Vergleich der beiden Scores schätzen die Studierenden ihr Wissen im Vergleich zum Können höher ein.

Diskussion: Die Studierenden schätzen nach der Lernendenstation ihr Wissen und Können insgesamt als höher ein. Das entspricht der Erwartung des Projektes, aber es fällt auf, dass die Items, die vor allem im Fokus des Projektes standen, nur geringe Veränderungen in der Selbsteinschätzung zeigen.

D. Wilborn $(\bowtie)$

Department Pflege und Management, Fakultät Wirtschaft und Soziales, Hochschule für Angewandte Wissenschaften Hamburg HAW, Hamburg, Deutschland

E-Mail: doris.wilborn@haw-hamburg.de 